\title{
Noninvasive measurement of intestinal epithelial damage at time of refeeding can predict clinical outcome after necrotizing enterocolitis
}

Kostan W. Reisinger', Joep P.M. Derikx', Geertje Thuijls', David C. van der Zee², Hens A.A. Brouwers ${ }^{3}$, Annemarie A. van Bijnen', Tim G.A.M. Wolfs ${ }^{4}$, L.W. Ernest van Heurn', Wim A. Buurman' and Boris W. Kramer ${ }^{4}$

BACKGROUND: Reintroduction of enteral nutrition in neonates with necrotizing enterocolitis (NEC) should take place when the gut is ready for its normal function. Too early a start of oral feeding might lead to disease relapse, whereas prolonged discontinuation of enteral nutrition is associated with impaired gut function and parenteral nutrition-related complications. This study evaluated whether noninvasive urinary measurement of intestinal fatty acid binding protein (I-FABP) at the time of refeeding can predict clinical outcome in neonates with NEC.

METHODS: Urinary I-FABP concentrations were measured in 21 infants with NEC just before reintroducing enteral nutrition. Poor outcome was defined as unsuccessful reintroduction of enteral feeding (EF), (re)operation for NEC, or death related to NEC after reintroduction of EF.

RESULTS: Median urinary I-FABP levels in neonates with poor outcome $(n=5)$ were significantly higher as compared with I-FABP levels in neonates with good outcome $(n=16)(P<0.01)$. A clinically significant cutoff value of $963 \mathrm{pg} / \mathrm{ml}$ was found to discriminate between infants with poor outcome and those with good outcome (sensitivity $80 \%$, specificity $94 \%$ ).

CONCLUSION: Noninvasive urinary I-FABP measurement at time of refeeding differentiates neonates with poor outcome from neonates with good outcome in NEC. Urinary I-FABP measurement may therefore be helpful in the timing of $\mathrm{EF}$ in neonates with NEC.

N ecrotizing enterocolitis (NEC) is a severe gastrointestinal disease with high morbidity and mortality $(20-40 \%)$, affecting predominantly premature neonates (1). The treatment of choice for infants suffering from mild to moderate NEC includes cardiorespiratory support if necessary, broad-spectrum antibiotics, and discontinuation of enteral feeding (EF) (1-3). Surgical intervention is considered in patients with a perforation or advanced NEC that does not improve with conservative treatment $(2,4)$.

After the initial treatment of NEC, there is no common opinion regarding the time when $\mathrm{EF}$ can be reintroduced. There are no clinical, radiological, or laboratory parameters to determine when refeeding of the infant is safe. Refeeding at a very early stage has been suggested to lead to recurrence of the disease (5), whereas prolonged discontinuation of EF can worsen gut function (6) and impair growth. Moreover, prolonged use of parenteral nutrition is associated with catheter-related septicemia and longer duration of hospital stay (7).

In a recent study, we demonstrated that urinary intestinal fatty acid binding protein (I-FABP) is a suitable noninvasive marker to confirm the diagnosis of NEC in patients with early symptoms of NEC (8). I-FABP, a small $(14-15 \mathrm{kDa})$ cytosolic protein, is solely present in mature epithelial cells of the small and large intestine (9). It is immediately released into the circulation upon loss of cell membrane integrity and passes the glomerular filter with a fractional renal excretion of $28 \%$ and a half-life of $11 \mathrm{~min}$, which makes it detectable in the urine (10). Therefore, urinary I-FABP levels accurately reflect the actual extent of intestinal epithelial cell damage, which makes I-FABP an indicator of disease progression (8). Although initially elevated, urinary I-FABP levels decrease when disease severity diminishes (11). We therefore hypothesized that I-FABP levels may be useful to determine the ideal time for reintroducing EF.

In this study, we measured urinary I-FABP concentrations in infants who were previously treated for NEC at the time oral feeding was introduced and evaluated the outcome of these children.

\section{RESULTS}

\section{Patients and Outcome}

Twenty-one neonates were included in the study, of whom 12 were males. Patient characteristics are listed in Table 1. There were no significant differences between groups in gestational age, birth weight, sex, or in blood laboratory tests (C-reactive protein, leukocytes, and platelets) at time of refeeding (Table 1).

Five of the 21 infants with NEC had a poor clinical outcome, and reintroduction of EF was unsuccessful (total parenteral nutrition was restarted). Four of these five received an operation

\footnotetext{
'Department of Surgery, Maastricht University Medical Center, and Nutrition and Toxicology Research Institute (NUTRIM), Maastricht, The Netherlands; ${ }^{2}$ Department of Surgery, Wilhelmina Children's Hospital, University Medical Center, Utrecht, The Netherlands; ${ }^{3}$ Department of Neonatology, Wilhelmina Children's Hospital, University Medical Center, Utrecht, The Netherlands; ${ }^{4}$ Department of Pediatrics, Maastricht University Medical Center, and School for Oncology and Developmental Biology (GROW), Maastricht, The Netherlands. Correspondence: Kostan W. Reisinger (k.reisinger@maastrichtuniversity.nl)
} 
Table 1. Baseline characteristics and conventional laboratory tests

\begin{tabular}{lcccc}
\hline & & Good & & \\
& Poor outcome & outcome & Total & $P$ \\
\hline $\begin{array}{l}\text { Gestational } \\
\text { age (days) }\end{array}$ & 207 & 224 & 224 & 0.55 \\
Birth weight & 1,300 & 1,463 & 1,460 & 0.78 \\
(g) & $(1,185-2,960)$ & $(860-2,106)$ & $(860-2,960)$ & \\
Sex & $3 \mathrm{M} 2 \mathrm{~F}$ & $9 \mathrm{M} 7 \mathrm{~F}$ & $12 \mathrm{M} 9 \mathrm{~F}$ & 1.00 \\
Surgery before & $3 / 5$ & $6 / 16$ & $9 / 21$ & 0.61 \\
refeeding & $(60 \%)$ & $(37.5 \%)$ & $(42.9 \%)$ & \\
CRP (mg/l) & 1.8 & 40 & 5.8 & 0.11 \\
& $(1.0-5.8)$ & $(1.0-93)$ & $(1.0-93)$ & \\
Leukocytes & 14.5 & 10.1 & 10.4 & 0.16 \\
$\left(\times 10^{9}\right.$ cells/l) & $(10.3-16.5)$ & $(8.0-20.1)$ & $(8.0-20.1)$ & \\
Platelets & 309 & 120 & 191.5 & 0.11 \\
$\left(\times 10^{9}\right.$ cells/l) & $(96-892)$ & $(29-296)$ & $(29-892)$ & \\
\hline
\end{tabular}

Data are presented as median (range).

CRP, $C$-reactive protein; $F$, female; $M$, male.

or reoperation on NEC within $30 \mathrm{~d}$ after reintroduction of EF, and the fifth child died within $30 \mathrm{~d}$ after reintroduction of oral feeding (Table 2). Three of the four infants operated for NEC after refeeding had been operated on earlier, $0-2 \mathrm{~d}$ after the initial diagnosis. In all four cases, NEC was the indication for operation. This was confirmed by histopathology. The other 16 neonates recovered from NEC after EF was restarted, without the need for surgery (group with a good clinical outcome).

The median period of time between birth and NEC diagnosis was 9 (4-30) $d$ in the good outcome group and 5 (2-18) $\mathrm{d}$ in the poor outcome group, $P=0.28$. The median period of time after NEC diagnosis that infants received no feeding by mouth was 9 (6-19) $d$ in the group with good outcome and $10(6-14) \mathrm{d}$ in the group with poor outcome, $P=0.50$. In the poor outcome group, the period of refeeding lasted for 8 (3-34) d. These results are shown in Table 3. The median period of time until full EF was achieved was $9 \mathrm{~d}$ (no difference between groups).

\section{High Urinary I-FABP Levels at Time of Refeeding Correlates With Poor Outcome}

Median urinary I-FABP:creatinine ratio (I-FABP corrected for urine concentration) just before reintroduction of EF was significantly higher in neonates with poor outcome (6.00 (0.53247.10) $\mathrm{pg} / \mathrm{nmol}$ creatinine) as compared with neonates with good outcome (0.38 (0.03-1.41) pg/nmol creatinine), $P<0.01$. To distinguish neonates with poor outcome from neonates with good outcome, an ideal cutoff value of $2.23 \mathrm{pg} / \mathrm{nmol} \mathrm{crea-}$ tinine was found with a sensitivity of $80 \%$ and a specificity of $100 \%$. This resulted in a positive likelihood ratio (LR) of $>12.8$ and a negative LR of 0.2 .

Similar data were obtained when I-FABP was not corrected for urine concentration (Figure 1). This analysis led to an ideal cutoff value of $963 \mathrm{pg} / \mathrm{ml}$, with a sensitivity of $80 \%$ and a specificity of $94 \%$, and a positive LR of 12.8 and a negative LR of 0.21 . The area under the curve for urinary I-FABP in
Table 2. Specification of patients with poor clinical outcome

\begin{tabular}{lccc}
\hline & $\begin{array}{c}\text { Discontinuation } \\
\text { of enteral feeding } \\
\text { after refeeding }\end{array}$ & $\begin{array}{c}\text { Operation after } \\
\text { refeeding }\end{array}$ & $\begin{array}{c}\text { Death after } \\
\text { refeeding }\end{array}$ \\
\hline Patient A & Yes & Yes $^{\mathrm{a}}$ & No \\
Patient B & Yes & No & Yes $^{\mathrm{b}}$ \\
Patient C & Yes & Yes & Yes $^{\mathrm{c}}$ \\
Patient D & Yes & Yes $^{\mathrm{a}}$ & Yes $^{\mathrm{d}}$ \\
Patient E & Yes & Yes $^{\mathrm{a}}$ & No \\
\hline
\end{tabular}

Columns are ordered from left to right by increasing severity of adverse outcome. NEC, necrotizing enterocolitis.

aSecond operation for NEC. Active NEC was found in all cases. ${ }^{b}$ Died within $30 \mathrm{~d}$ after diagnosis of NEC. 'Died $71 \mathrm{~d}$ after diagnosis of NEC; cause of death: persistent neonatal seizures. ${ }^{~}$ Died $162 \mathrm{~d}$ after diagnosis of NEC; cause of death: treatment discontinued because of severe neurological damage.

Table 3. Time course

\begin{tabular}{lrccc}
\hline & $\begin{array}{c}\text { Poor } \\
\text { outcome }\end{array}$ & $\begin{array}{c}\text { Good } \\
\text { outcome }\end{array}$ & Total & $P$ \\
\hline $\begin{array}{l}\text { Diagnosis at birth (days) } \\
\begin{array}{l}\text { Diagnosis after refeeding } \\
\text { (days) }\end{array}\end{array}$ & $\begin{array}{l}\text { 10(2-18) } \\
\text { Refeeding after the }\end{array}$ & $9(4-30)$ & $9(2-30)$ & 0.28 \\
$\begin{array}{l}\text { Recond discontinuation } \\
\text { of enteral feeding (days) }\end{array}$ & $8(3-34)$ & $9(6-19)$ & 0.50 \\
\hline
\end{tabular}

Data are presented as median (range).

differentiating neonates with poor outcome from neonates with good outcome was 0.93 (95\% confidence interval 0.78 1.00) for I-FABP corrected for urine concentration and 0.95 (95\% confidence interval 0.86-1.00) for I-FABP not corrected for urine concentration. Median urinary I-FABP for 20 premature neonates without gastrointestinal symptoms (matched controls) was 358 (174-924) pg/ml (Figure 1).

Relative Difference in Urinary I-FABP Levels From Diagnosis to Time of Refeeding as a Predictor of Poor Outcome

Another approach to predicting the outcome of reintroduction of EF would be to measure the relative reduction of intestinal damage as reflected by the differences in I-FABP levels from the moment of diagnosis to the moment of oral refeeding. The relative difference $(\Delta \mathrm{Rel})$ in urinary I-FABP from diagnosis (Diag) to reintroduction of EF was calculated as follows:

$$
\Delta \mathrm{Rel}_{\text {Diag-EF }}=\left((\mathrm{I}-\mathrm{FABP})_{\mathrm{EF}}-(\mathrm{I}-\mathrm{FABP})_{\text {Diag }}\right) /(\mathrm{I}-\mathrm{FABP})_{\text {Diag }} \times 100 \%,
$$

where (I-FABP) is the concentration of I-FABP (not corrected for urine concentration) $(\mathrm{pg} / \mathrm{ml}) . \Delta \mathrm{Rel}_{\text {Diag-EF }}$ in neonates with good outcome $(-100 \%(-100 \%$ to $-22 \%))$ was significantly lower than $\Delta \operatorname{Rel}_{\text {Diag-EF }}$ in neonates with poor outcome $(-66 \%(-87 \%$ to $+1137 \%)), P<0.05$ (Figure 2). To distinguish neonates with poor outcome from neonates with good outcome, an ideal cutoff value of $-90 \%$ was found with a sensitivity of $100 \%$ and a specificity of $75 \%$. This resulted in a positive LR of 4.00 and a negative LR of 0.00 . The area under the curve 


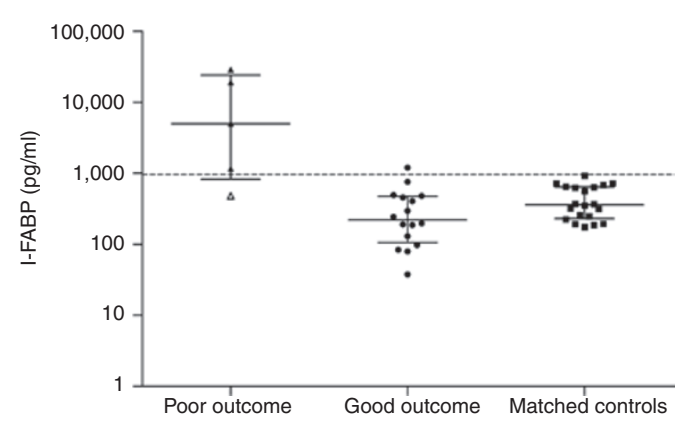

Figure 1. Urinary intestinal fatty acid binding protein (I-FABP) levels of infants on the day of reintroduction of enteral feeding, median $9 \mathrm{~d}$ after diagnosis of necrotizing enterocolitis. Data are expressed as median I-FABP levels with interquartile ranges. A cutoff value of $963 \mathrm{pg} / \mathrm{ml}$ is marked by the dotted line. I-FABP levels of controls matched for gestational age are depicted. Black triangles, infants operated on after refeeding; black circles, infants not operated on after refeeding; $\Delta$ symbol, died after refeeding; black squares, matched controls.

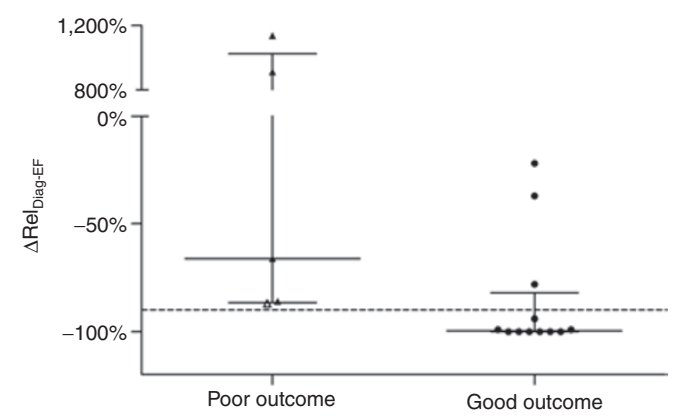

Figure 2. Relative differences with median and interquartile ranges of urinary intestinal fatty acid binding protein from diagnosis to reintroduction of enteral feeding $\left(\Delta \mathrm{Rel}_{\text {Diag-EF }}\right.$ ). A cutoff value of $-90 \%$ is marked by the dotted line. Black triangles, infants operated on after refeeding; black circles, infants not operated on after refeeding; $\Delta$ symbol, died after refeeding.

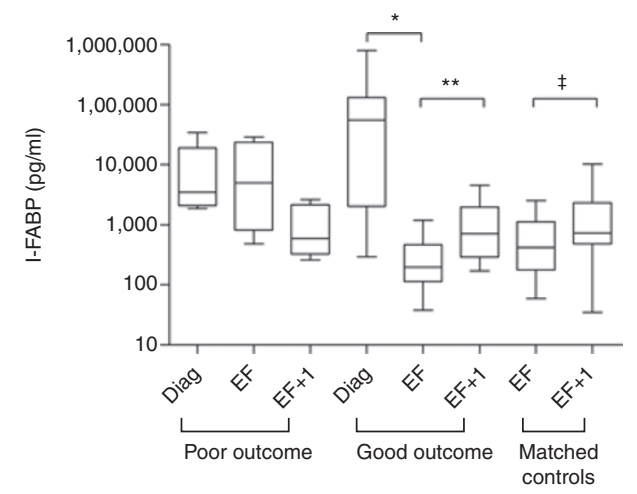

Figure 3. Urinary intestinal fatty acid binding protein levels on the day of diagnosis (Diag), the day of reintroduction of enteral feeding (EF), and $1 \mathrm{~d}$ after reintroduction of EF. ${ }^{*} P=0.002 ;{ }^{*} P=0.04 ;{ }^{\ddagger} P=0.03$. I-FABP, intestinal fatty acid binding protein.

for $\Delta \operatorname{Rel}_{\text {Diag-EF }}$ in differentiating neonates with poor outcome from neonates with good outcome was 0.87 (95\% confidence interval 0.69-1.00) (receiver operating characteristic curve not shown). Actual urinary I-FABP levels at the time of diagnosis are depicted in Figure 3. There was no significant difference in urinary I-FABP levels at the time of diagnosis between the groups. In conclusion, the former analysis based on actual values at the moment of refeeding produced better data.

\section{Kinetics of I-FABP Following Reintroduction of EF}

In the analyses, both I-FABP corrected and uncorrected for urine concentration were used. Next, we analyzed whether differences could be detected between neonates with good clinical outcome and those with poor clinical outcome early during feeding. To this end, we compared data at EF with data on $\mathrm{EF}+1$. Analysis of the data revealed a significant increase in urinary I-FABP from EF (0.38 (0.03-1.41) pg/nmol creatinine) to EF+1 $(1.41(0.53-2.74) \mathrm{pg} / \mathrm{nmol}$ creatinine, $P<0.05)$ in the neonates with good outcome. In contrast, neonates with poor outcome showed no increase from $\mathrm{EF}$ to $\mathrm{EF}+1$.

Similar data were obtained in the case in which I-FABP was not corrected for urine concentration; a significant increase in urinary I-FABP from EF $(197(37.7-1,196) \mathrm{pg} / \mathrm{ml})$ to $\mathrm{EF}+1(712(170-4,537) \mathrm{pg} / \mathrm{ml}, P=0.04)$ was observed in the group with good outcome (Figure 3 ). This increase (EF, 421 (59-2,553); EF+1, 733 (35-10,278), $P=0.03$, Figure 3) was also observed in the control group of nine infants without intestinal injury in whom feeding was discontinued and reintroduced, indicating a physiological rather than a pathological response.

\section{DISCUSSION}

One of the challenges in the treatment of the infant with NEC is to choose the best moment to reintroduce EF. Although no benefit has been shown for delayed (12) or slow (13) introduction of EF in the prevention of NEC, evidence is lacking on the length of the period between the treatment of NEC and the moment at which EF can be restarted safely. Although a period of 7-10 d of discontinuation of EF has been recommended (14), faster reintroduction after NEC seems feasible in some cases. In a study by Bohnhorst et al. (7), EF was restarted as early as $4 \mathrm{~d}$ after diagnosis of NEC when portal vein gas was absent on ultrasound. These infants showed less catheter-related sepsis and a shorter hospital stay as compared with historical controls in which EF was reinitiated on day 10 (7). Moreover, return to EF may reduce the development of villus atrophy (15). We consider that daily measurement of urinary I-FABP could help to identify infants who can be safely returned to oral feeding.

The results of this study show that urinary I-FABP is a suitable noninvasive marker to determine which infants with NEC in whom EF is restarted are at risk for a poor outcome, in terms of mortality or reoperation. In addition to clinical assessment, urinary I-FABP measurement can therefore be a helpful tool for the neonatologist in deciding which infants can be refed safely in the course of NEC, especially when clinical improvement is doubted. At the moment, no laboratory markers exist to confirm this decision. In the population studied, a cutoff value at the time of oral refeeding of $963 \mathrm{pg} / \mathrm{ml}$ could discriminate neonates with poor outcome from those with good outcome, with a sensitivity of $80 \%$ and a specificity of $94 \%$. Whether clinical deterioration 
could have been avoided by delaying reintroduction of EF, or whether clinical deterioration can directly be caused by onset of refeeding cannot be determined by these data. Further study in a large number of patients is needed to evaluate the cutoff value of $963 \mathrm{pg} / \mathrm{ml}$ and its clinical usefulness.

Remarkably, I-FABP levels increased early during feeding in the group with good outcome and in a control group without NEC or other severe intestinal injury. This may be a result of a physiological response of the gut when challenged by EF. In a piglet model, rapid enterocyte turnover and mucosal structural changes were observed $8 \mathrm{~h}$ after introduction of EF (16). Unexpectedly, I-FABP levels seemed to decrease early during refeeding in the group with poor outcome; however, the difference was not statistically significant. This could be a consequence of the reduction of enterocyte mass as described earlier in severe mucositis induced by myeloablative conditioning (17). However, the size of this group is relatively small, which is an inevitable problem, given that many of the children who die of NEC die shortly after NEC diagnosis without the possibility to restart EF.

\section{Conclusion}

In conclusion, when I-FABP levels were $>963 \mathrm{pg} / \mathrm{ml}$ following clinical progression that resulted in reintroducing $\mathrm{EF}$, there was a high chance of clinical deterioration in this NEC population. Urinary I-FABP measurement may therefore be helpful in the timing of EF in neonates with NEC. However, this hypothesis has to be validated in a prospective trial, with different treatment strategies determined on the basis of urinary I-FABP measurement that serves as an addition to clinical judgment.

\section{METHODS}

\section{Patients, Feeding Protocol, and Sample Collection}

Twenty-one consecutive infants, in whom EF was reintroduced after treatment for NEC in the neonatal intensive care units at Maastricht University Medical Centre and Wilhelmina Children's Hospital in Utrecht, between July 2005 and August 2010, were included in the study. NEC was diagnosed with the current gold standard of abdominal X-ray showing pneumatosis intestinalis (Bell stage II or higher) (2). Twenty consecutive premature infants without gastrointestinal symptoms (matched with NEC infants for gestational age at birth) admitted to the neonatal intensive care unit at Maastricht University Medical Centre were also included for the evaluation of control values. Indication for admission into the neonatal intensive care unit was small for gestational age or prematurity in all cases. In these premature neonates serving as controls, a urine sample was collected. A second control group of nine neonates without intestinal injury was included, in whom EF was stopped and restarted. Indications for cessation of EFs were suspicion of NEC (eventually diagnosed as sepsis) or laparotomy for malformation or atresia without significant intestinal epithelial cell damage. Written consent was obtained from both parents, and the study was conducted with the approval of the local medical ethical committees and according to the revised version of the Declaration of Helsinki (October 2008, Seoul). The principles of good clinical practice were followed during this study.

Feeding was restarted in patients who improved clinically: spontaneously breathing patients without intestinal pneumatosis on abdominal X-ray, without abdominal distention, and with peristalsis and a soft abdomen. This decision was made by the attending neonatologist, who was independent of the investigators. EF consisted of formula feeding, breast milk, or a combination of both and was increased with a maximum of $20 \mathrm{ml} / \mathrm{kg} /$ day.
Urine was obtained daily by placing a dental cotton roll (Henry Schein, Almere, The Netherlands) in the diaper of the neonate. Once the roll was filled with urine, it was placed into a sterile 5-ml syringe (Becton Dickinson, Oxford, UK), and the urine was pressed into Micronic tubes (Micronic B.V., Lelystad, The Netherlands) and stored at $-20^{\circ} \mathrm{C}$ until analysis. Urine samples were collected at the time of diagnosis (Diag), just before reintroduction of EF, and the day after reintroduction of $\mathrm{EF}(\mathrm{EF}+1)$.

All patient materials were collected in a prospective manner; however, the analyses were performed by one person who was blinded to clinical diagnoses after completion of patient inclusion. Attending clinicians were therefore not aware of I-FABP levels when deciding to restart EF.

\section{Urinary I-FABP Measurement}

Urinary I-FABP was measured using an in-house enzyme-linked immunosorbent assay that selectively detects human I-FABP (standard: $10-1,280 \mathrm{pg} / \mathrm{ml})$. Values are expressed as concentration $(\mathrm{pg} / \mathrm{ml})$ and ratio $(\mathrm{pg} / \mathrm{nmol}$ creatinine) of I-FABP $(\mathrm{pg} / \mathrm{ml})$ to creatinine $(\mu \mathrm{mol} / \mathrm{l})$, to compensate for variations in urine concentration.

\section{Statistical Analyses}

Normality was tested by the Kolmogorov-Smirnov test. The MannWhitney $U$ test was used for between-groups comparison for continuous data. Dichotomous variables were compared using Fisher's exact test. All data are presented as median and range. Outcome was defined as poor clinical outcome (unsuccessful introduction of EF (i.e., restarting total parenteral nutrition), (re)operation, or death within $30 \mathrm{~d}$ after diagnosis of NEC) or good clinical outcome. To find a cutoff point of urinary I-FABP levels at the time of refeeding that most accurately predicts outcome, a receiver operating characteristic curve was drawn by plotting sensitivity against 1-specificity for all possible thresholds. Overall accuracy of urinary I-FABP to predict outcome was represented by the area under the curve. The best cutoff point was defined as the cutoff point with the maximum sum of sensitivity and specificity, after taking the range of normal values into account. Statistical analyses were performed with Prism 5.0 for Windows (GraphPad Software, San Diego, CA). STARD statement for reporting studies of diagnostic accuracy was used in this study (18).

\section{STATEMENT OF FINANCIAL SUPPORT}

There are no financial relationships relevant to this article to disclose.

Disclosure: The authors declare no conflict of interest.

\section{REFERENCES}

1. Lin PW, Stoll BJ. Necrotising enterocolitis. Lancet 2006;368:1271-83.

2. Bell MJ, Ternberg JL, Feigin RD, et al. Neonatal necrotizing enterocolitis. Therapeutic decisions based upon clinical staging. Ann Surg 1978;187:1-7.

3. Neu J. Neonatal necrotizing enterocolitis: an update. Acta Paediatr Suppl 2005;94:100-5.

4. Lee JS, Polin RA. Treatment and prevention of necrotizing enterocolitis. Semin Neonatol 2003;8:449-59.

5. Berseth CL. Feeding strategies and necrotizing enterocolitis. Curr Opin Pediatr 2005;17:170-3.

6. Lin PW, Nasr TR, Stoll BJ. Necrotizing enterocolitis: recent scientific advances in pathophysiology and prevention. Semin Perinatol 2008;32:70-82.

7. Bohnhorst B, Müller S, Dördelmann M, Peter CS, Petersen C, Poets CF. Early feeding after necrotizing enterocolitis in preterm infants. J Pediatr 2003;143:484-7.

8. Thuijls G, Derikx JP, van Wijck K, et al. Non-invasive markers for early diagnosis and determination of the severity of necrotizing enterocolitis. Ann Surg 2010;251:1174-80.

9. Veerkamp JH, Paulussen RJ, Peeters RA, Maatman RG, van Moerkerk HT, van Kuppevelt TH. Detection, tissue distribution and (sub)cellular localization of fatty acid-binding protein types. Mol Cell Biochem 1990;98:11-8.

10. van de Poll MC, Derikx JP, Buurman WA, et al. Liver manipulation causes hepatocyte injury and precedes systemic inflammation in patients undergoing liver resection. World J Surg 2007;31:2033-8. 
11. Evennett NJ, Hall NJ, Pierro A, Eaton S. Urinary intestinal fatty acid-binding protein concentration predicts extent of disease in necrotizing enterocolitis. J Pediatr Surg 2010;45:735-40.

12. Morgan J, Young L, McGuire W. Delayed introduction of progressive enteral feeds to prevent necrotising enterocolitis in very low birth weight infants. Cochrane Database Syst Rev 2011: CD001970

13. Morgan J, Young L, McGuire W. Slow advancement of enteral feed volumes to prevent necrotising enterocolitis in very low birth weight infants. Cochrane Database Syst Rev 2011:CD001241.

14. Neu J, Walker WA. Necrotizing enterocolitis. N Engl J Med 2011;364: 255-64.
15. Kliegman RM, Walker WA, Yolken RH. Necrotizing enterocolitis: research agenda for a disease of unknown etiology and pathogenesis. Pediatr Res 1993;34:701-8.

16. Oste M, Van Haver E, Thymann T, Sangild P, Weyns A, Van Ginneken CJ. Formula induces intestinal apoptosis in preterm pigs within a few hours of feeding. JPEN J Parenter Enteral Nutr 2010;34:271-9.

17. Derikx JP, Blijlevens NM, Donnelly JP, et al. Loss of enterocyte mass is accompanied by diminished turnover of enterocytes after myeloablative therapy in haematopoietic stem-cell transplant recipients. Ann Oncol 2009;20:337-42.

18. Bossuyt PM, Reitsma JB, Bruns DE, et al. The STARD statement for reporting studies of diagnostic accuracy: explanation and elaboration. Clin Chem 2003;49:7-18. 